\title{
Evaluación de la prueba de inmunofluorescencia indirecta para el diagnóstico de leptospirosis humana
}

\author{
Piedad Agudelo-Flórez, Marcos Restrepo, María Amparo Lotero \\ Instituto Colombiano de Medicina Tropical, Instituto de Ciencias de la Salud (ICMT-CES), Sabaneta, Colombia.
}

Introducción. El diagnóstico de la leptospirosis es difícil debido al amplio espectro de síntomas clínicos que presenta. Varias pruebas diagnósticas serológicas se han desarrollado, pero su aplicación y utilidad en Colombia no han sido determinadas.

Objetivos. Evaluar la prueba de inmunofluorescencia indirecta para el diagnóstico de leptospirosis humana y determinar así los anticuerpos de las clases inmunoglobulina $\mathrm{G}$ y $\mathrm{M}$ producidos contra Leptospira.

Materiales y métodos. Se establecieron tres grupos de estudio. El primero incluyó 19 muestras positivas para leptospirosis de pacientes con diagnóstico clínico y prueba de microaglutinación positiva; el segundo, 40 muestras de personas sin antecedentes de leptospirosis y con microaglutinación negativa, y el tercero con 96 muestras de pacientes con otras enfermedades diferentes a leptospirosis. Todas las muestras fueron procesadas por inmunofluorescencia indirecta.

Resultados. Se determinó que la inmunofluorescencia indirecta tiene sensibilidad de $89,47 \%$, especificidad de $100 \%$, valor predictivo negativo de 95,2\% (IC95\%82,6 a 99,2) y valor predictivo positivo de $100 \%$. En forma paralela, un estudio exploratorio en 27 muestras de suero que habían sido remitidas para diagnóstico de diferentes síndromes febriles encontró que $11 \%$ de ellas fueron positivas por inmunofluorescencia indirecta para anticuerpos IgM contra Leptospira. Conclusiones. La inmunofluorescencia indirecta es una alternativa que complementa el diagnóstico de leptospirosis y los estudios seroepidemiológicos. La presencia de anticuerpos dirigidos contra Leptospira en las muestras de síndrome febril del estudio indica que la leptospirosis es una de las causas de este síndrome que el clínico debe explorar.

Palabras clave: Leptospira, leptospirosis, enfermedad de Weil, anticuerpos, serología, técnica del anticuerpo fluorescente indirecta.

\section{Evaluation of Indirect Inmunofluorescence assay for diagnosis of human leptospirosis}

Introduction. The diagnosis of leptospirosis is difficult because it presents an extremely broad spectrum of symptoms. A number of diagnostic serological assays have been developed, but their performance and utility have not been evaluated in Colombia.

Objectives. To evaluate the IgM-IgG-indirect inmunofluorescence assay to detect antibodies against Leptospira in human serum samples and to diagnose leptospirosis.

Materials and methods. A panel of 19 sera from leptospirosis cases with clinical diagnosis and positive microscopic agglutination test, 40 samples from individuals without history of leptospirosis and negative microscopic agglutination test, and 96 samples from patients with other infectious diseases were evaluated.

Results. The sensitivity of the indirect inmunofluorescence assay was $89.47 \%$, its specificity was $100 \%$, the negative predictive value was 95.2\% ( $\mathrm{Cl} 95 \% 82.6-99.2)$, and its positive predictive value was $100 \%$. At the same time an exploratory study of 27 samples from different febrile syndromes, $11 \%$ were positive by IgM-indirect inmunofluorescence assay against Leptospira.

Conclusions. The data indicate that indirect inmunofluorescence assay is useful as a diagnostic adjunct to clinical diagnosis and for seroepidemiologic studies. The presence of IgM antibodies against Leptospira in sera from cases of febrile syndrome indicate that leptospirosis is one of the causes of this syndrome and should be considered by the clinician. 
Keywords: Leptospira, leptospirosis, Weil disease, antibodies, serology, indirect fluorescent antibody technique.

La leptospirosis es una zoonosis reemergente de gran incidencia en regiones tropicales debido a factores ambientales, climáticos y sociales que favorecen la transmisión. El agente etiológico es Leptospira spp, bacteria que puede infectar a la mayoría de especies de mamíferos cuando entran en contacto directo o indirecto con agua o suelo contaminado con orina de animales infectados (1-3). Mediante métodos moleculares se han definido varias especies de Leptospira patógenas: Leptospira alexanderi, Leptospira interrogans, Leptospira fainei, Leptospira inadai, Leptospira borgpetersenii, Leptospira santarosai, Leptospira weilii, Leptospira meyeri, Leptospira noguchii, Leptospira wolbachii, Leptospira alstoni, Leptospira kirschneri, Leptospira parva y las genoespecies 1, 3, 4 y 5. Las leptospiras saprófitas se agruparon como Leptospira biflexa, con cinco serovariedades clasificadas, además de 49 serovariedades de Leptospira sp sin definición de la especie a la que pertenecen (4-6).

En los seres humanos la infección ocurre en forma esporádica o en brotes epidémicos. Cuando causa enfermedad se presenta como un síndrome febril agudo con manifestaciones clínicas variadas. Para llegar a su diagnóstico definitivo se requiere tener en cuenta los antecedentes epidemiológicos, la presencia de anticuerpos y en algunos casos, el aislamiento del microorganismo $(1,7)$. El amplio espectro de síntomas clínicos dificulta el diagnóstico rápido, por lo que el curso de la enfermedad puede variar rápidamente de las formas intermedias a las fatales. Es necesario disponer de un diagnóstico oportuno para facilitar el manejo del paciente y el inicio de la terapia correspondiente.

En la fase aguda de la enfermedad, durante la respuesta febril, las leptospiras pueden ser aisladas en medio de cultivo semisólido e incluso

Correspondencia:

Piedad Agudelo-Flórez

Cra 43A No 52 Sur-99, Sabaneta-Colombia, AA 52162

Teléfono 574-3053500, fax 574-3014258;pagudelo@ces.edu.co

Recibido: 09/09/05; aceptado: 27/03/06 visualizadas al microscopio en muestras de sangre con objetivo de campo oscuro. Este último método presenta inconvenientes cuando el número de bacterias en sangre es muy bajo, la proporción de aislamiento bacteriano es reducida y se pueden confundir las verdaderas leptospiras con cadenas proteínicas de la sangre anticoagulada (seudoespiroquetas) (8); estos factores de error obligan a que toda observación directa de leptospiras deba ser confirmada siempre con un cultivo positivo, lo que implica esperar varias semanas para entregar un diagnóstico confiable.

Con respecto a las pruebas serológicas utilizadas para el diagnóstico de la leptospirosis, la microaglutinación en campo oscuro (MAT) es la prueba de referencia de la Organización Mundial de la Salud (OMS) (1) y está disponible en laboratorios especializados, pero presenta una sensibilidad baja, requiere sueros pareados para la detección de la seroconversión, muestra reacciones cruzadas entre serogrupos y consume mucho reactivo, ya que es necesario mantener cultivos en repique constante y probar la mayor cantidad de serovariedades posibles, sin olvidar, además, que la utilización de leptospiras vivas presenta riesgo ocupacional de infección para el personal de laboratorio (9-12).

En los últimos años se han desarrollado otras pruebas serológicas para el diagnóstico de la leptospirosis, pero su aplicación, evaluación y validación para Colombia no se ha establecido (9-19). En esta publicación se informa sobre la evaluación de una prueba de inmunofluorescencia indirecta (IFI) para detectar anticuerpos IgM e lgG que orienten el diagnóstico de la leptospirosis y que tiene aplicación para estudios epidemiológicos en gran escala.

\section{Materiales y métodos \\ Cultivos de Leptospira spp}

El cepario correspondiente a Leptospira spp se mantuvo en cultivos continuos en medio semisólido de Fletcher (Difco) y en medio líquido EMJH (Elling-hausen-McCullough-Johnson-Harris- 
Becton-Dickinson-Biosciences) suplementado con $10 \%$ de medio de enriquecimiento comercial (Becton-Dickinson-Biosciences).

El cepario está constituido por ocho serovariedades de Leptospira spp entre las que se cuentan, L. interrogans Copenhageni cepa M20, $L$.interrogans Icterohaemorrhagiae cepa RGA $L$ .interrogans Pomona cepa Pomona, L. interrogans Hardjo cepa Hardjoprajitno, L. interrogans Valbuzzi cepa Valbuzzi, $L$.interrogans Canicola cepa Hond Utrecht IV, L. borgpeterseneii Bratislava cepa Jez Bratislava y L. biflexa Semaranga cepa Patoc 1. Estas cepas fueron donadas por el Instituto Colombiano Agropecuario (ICA) en Bogotá, y algunas por el Laboratorio de Leptospirosis y Borreliosis de la Universidad Nova de Lisboa, Portugal.

La evaluación de la prueba de IFI determinó la sensibilidad, especificidad y valores predictivos de la reacción, empleando como prueba de oro el MAT. Se constituyeron cuatro grupos de estudio. El número de muestras de suero o de plasma a estudiar en cada uno de los grupos se describe a continuación.

a) Para el estudio de la sensibilidad, el grupo estuvo constituido por el total de los niños o adultos de Medellín y Apartadó (Antioquia) que consultaron a las unidades de salud durante el período de estudio (1999-2001) por síndrome febril compatible clínicamente con leptospirosis, algunos de ellos con ictericia y todos con antecedentes epidemiológicos de riesgo para leptospirosis y que tuvieran prueba de MAT positiva con título igual o mayor a 1:200. Estos criterios son consistentes con registros bibliográficos sobre el tema $(1,20-22)$. Con base en estos criterios, se conformó un grupo de 19 muestras de sueros positivos así: 13 casos con muestras pareadas tomadas en fase aguda $y$ convaleciente con seroconversión y 6 casos con títulos mayores de 1:200 en una sola muestra tomada en fase convaleciente, uno de estos casos, además, con cultivo positivo para Leptospira sp. Para determinar que este aislamiento pertenecía a una cepa patógena, el crecimiento se realizó a $13^{\circ} \mathrm{C}$ en la presencia de 8-azaguanine según lo recomendado por la OMS (1). b) La muestra para la evaluación de la especificidad se estableció por conveniencia, quedando constituido este grupo por 40 muestras de suero procedentes de voluntarios sanos sin antecedentes clínicos ni epidemiológicos de leptospirosis y con MAT negativa

c) La muestra para la evaluación de reacciones cruzadas se estableció por conveniencia, quedando constituido este grupo por 96 muestras de suero de pacientes con otras entidades clínicas diferentes a la leptospirosis y con MAT negativa.

d) Veintisiete muestras de plasma o suero de pacientes con síndrome febril no malárico y que fueron enviadas para diagnóstico de leptospirosis.

Se utilizaron sueros controles positivos donados por el Laboratorio de Leptospirosis y Borreliosis del Instituto de Medicina Tropical, Universidad Nova de Lisboa, Portugal. Las muestras negativas se obtuvieron de personas sin antecedentes clínicos ni epidemiológicos de leptospirosis. Los sueros positivos para otras enfermedades diferentes a leptospirosis se obtuvieron del Banco de Sueros del Instituto Colombiano de Medicina Tropical-CES (ICMT-CES). En este grupo se incluyeron muestras de infecciones tales como malaria, hepatitis $B$, hepatitis $A$, factor reumatoideo, salmonelosis, dengue y sífilis, esta última con prueba de FTA-ABS reactiva.

\section{Procedimientos serológicos}

\section{Prueba de microaglutinación (MAT)}

Todas las muestras fueron procesadas por MAT para detectar anticuerpos anti-Leptospira. Los antígenos fueron las cepas mantenidas en cultivo continuo en medio líquido EMJH transferidas cada siete días. Se incubaron a temperaturas promedio de $26^{\circ} \mathrm{C}$ a $30^{\circ} \mathrm{C}$. Cuando alcanzaron un crecimiento con una turbidez equivalente al 0,5 de la escala MacFarland, se utilizaron como antígenos para la prueba MAT.

La prueba se realizó según las recomendaciones de la OMS (1). Inicialmente se hizo un tamizaje de género de todas las muestras de suero a evaluar a una dilución de 1:50 con la cepa Patoc 1 de L. biflexa Semaranga, serovar Patoc. Se consideraron como negativas todas las muestras 
que no presentaron aglutinación del 50 al $75 \%$ de las leptospiras en este tamizaje. A las muestras que resultaron positivas se les hicieron diluciones al doble a partir de la dilución 1:50 y hasta 1:3.200, y se probaron con todas las demás serovariedades que constituyen el cepario. La lectura se hizo en microscopio de luz marca Nikon con condensador de campo oscuro y con aumento de 20X. El título de anticuerpos se fijó en la dilución inmediatamente anterior a aquella en la cual la reacción de aglutinación se vio negativa. Las pruebas se interpretaron como positivas cuando se observó aglutinación del 50 al $75 \%$ de las leptospiras con cualquiera de las serovariedades probadas en una dilución del suero mayor o igual a 1:100.

\section{Prueba de Inmunofluorescencia Indirecta (IFI)}

El antígeno utilizado para esta prueba se obtuvo de un cultivo de L. biflexa Semaranga, cepa Patoc 1 en medio líquido de tres a cuatro días de crecimiento. La reacción de IFI se estandarizó de acuerdo a la técnica de Appassakij et al. (13) y de la OMS (23) con algunas modificaciones. En términos generales la prueba se realizó de la siguiente forma: se depositaron $10 \mathrm{ul}$ del cultivo de leptospiras en cada uno de los círculos de los portaobjetos para inmunofluorescencia. Después de dejarse secar al aire, se fijaron las placas con acetona grado reactivo por 10 minutos, y luego estuvieron listas para ser usadas en la IFI o para guardarse a $-20^{\circ} \mathrm{C}$ hasta su uso.

Se hicieron diluciones al doble de los sueros a evaluar usando solución salina de fosfatos (PBS) $\mathrm{pH} 7,2$, desde $1: 10$ hasta 1:2.560, y se colocaron en cada círculo con el antígeno fijado. En cada prueba se procesaron sueros control positivo y negativo para leptospirosis. Después de una incubación en cámara húmeda a $37^{\circ} \mathrm{C}$ durante media hora, las placas se lavaron con PBS tres veces durante 10 minutos cada vez.

Se colocó en cada círculo conjugado antiinmunoglobulina G o M marcado con flouresceína (bioMérieux-Fluoline M [anti-cadenas $\mu$ ] y $G$ anticadenas g) y previamente titulado. Después de incubar y lavar en las condiciones señaladas, se procedió al montaje de las placas con glicerolPBS y se observó al microscopio de fluorescencia Nikon con un filtro de $495 \mathrm{~nm}$ y aumento de 40X.
Se determinó la positividad de las muestras comparándolas con los controles positivos y negativos y se definió el título positivo. El criterio para fijar el punto final del título del suero fue la más alta dilución que diera una lectura de fluorescencia comparada con el control positivo.

\section{Análisis estadístico}

El análisis estadístico se realizó con base en el registro de los datos en una tabla de doble entrada utilizando el programa EPIDAT 1.0 (SERGAS, Xunta de Galicia, Organización Panamericana de la Salud, OMS). De esta manera se calcularon los siguientes índices: con los resultados de los grupos "a" y "b" se realizaron los cálculos de la sensibilidad, la especificidad y los valores predictivos positivo y negativo de la prueba con sus correspondientes intervalos de confianza de 95\% (24). La comparación de los grupos se hizo mediante la prueba ji cuadrado. Se consideró un nivel de significación menor del $5 \%$. Con los resultados del grupo "c" se determinaron los títulos inespecíficos dados por otras etiologías diferentes a leptospirosis y por el factor reumatoideo positivo. Con los resultados del grupo "d" se realizó un estudio exploratorio de la frecuencia de leptospirosis en un grupo de pacientes con síndrome febril no malárico.

\section{Resultados}

El procesamiento del grupo de sueros positivos por la prueba de MAT determinó aglutinaciones con las ocho serovariedades de Leptospira spp en títulos mayores a 1:100 así: L. interrogans Valbuzzi con $67,34 \%$, L. interrogans Copenhageni con $63,2 \%$, L. interrogans Icterohaemorrhagiae con $40,81 \%$ L. interrogans Pomona con $42,85 \%$, L. interrogans Hardjo con 30,61\%, L. borgpeterseneii Bratislava con $26,53 \%$, L. interrogans Canicola y L. biflexa Semaranga cepa Patoc 1 cada una con $6,12 \%$. Se presentaron coaglutinaciones con más de tres serovares de Leptospira spp en 61,22\% de las muestras. Se consideró el serovar infectante como aquel en donde se registró el mayor título en una o en dos muestras pareadas. Las muestras que aglutinaron con L. biflexa lo hicieron en títulos mayores de 1:400 y, como no mostraron aglutinaciones significativas con ninguna de las otras especies probadas, se 
definieron como muestra positiva por "serovar no determinado".

Estos resultados mostraron que la respuesta de anticuerpos obtenida correspondía al género Leptospira, sin que pudiera definirse una especie ni una serovariedad prevalente de Leptospira en el grupo de sueros positivos. Con el fin de evaluar una prueba que usara el antígeno más representativo de todas las serovariedades registradas, se usó el antígeno de L. biflexa Semaranga patoc, que registra la respuesta de anticuerpos en su forma más amplia, o sea la respuesta géneroespecífica. Otro factor que contribuyó a esta decisión es que este antígeno es el recomendado por la OMS para la ejecución de la prueba.

El análisis estadístico de los grupos de estudio verdaderos positivos y verdaderos negativos, y con un antígeno de Leptospira género-específico, determinó que la IFI tuvo una sensibilidad para detectar el 89,47\% (IC95\%, 65,45 a 98,15) de los sueros del grupo de positivos. Los intervalos de confianza amplios se explican por el tamaño de la muestra. Por su parte, la prueba IFI detectó como negativas todas las muestras del grupo de verdaderos negativos, lo que equivale a una especificidad de $100 \%$. El valor predictivo negativo de la prueba fue de 95,2\% (IC95\% 82,6 a 99,2 ) y el valor predictivo positivo fue de $100 \%$.

Al procesar por IFI las muestras de los 19 pacientes con MAT positivo para leptospirosis se determinó que un individuo reaccionó sólo con lgM y otro sólo con IgG, y que 15 de los 19 pacientes fueron positivos por las dos inmunoglobulinas. Dos pacientes fueron negativos por IFI.

En el grupo de estudio conformado por sueros negativos para leptospirosis pero positivos para otras entidades, los cuales fueron utilizados para determinar reacciones cruzadas de la prueba de IFI y el punto de corte, se encontró inmunofluorescencia inespecífica en títulos menores o iguales a 1:40 en $\operatorname{lgG}$, y en títulos menores o iguales a 1:20 en la lgM; esto llevó a determinar que los niveles de $\operatorname{lgM}$ se presentan en título significativo a partir de 1:40 y que los niveles de IgG son significativos a partir de 1:80. En el cuadro 1 se presentan los resultados obtenidos en este grupo de estudio.
Cuadro 1. Determinación de reacciones cruzadas y punto de corte de la prueba de IFI IgG y M para el diagnóstico de leptospirosis humana.

\begin{tabular}{lccc}
\hline Grupo de estudio & $\mathbf{n}$ & $\begin{array}{c}\mathbf{I g M}^{\mathbf{a}} \\
\text { máximo } \\
\text { título (n) }\end{array}$ & $\begin{array}{c}\mathbf{I g G}^{\mathbf{b}} \\
\text { máximo } \\
\text { título (n) }\end{array}$ \\
\hline Malaria & 15 & $1: 20(1)$ & $1: 20(3)$ \\
Hepatitis B & 12 & $1: 20(6)$ & $1: 40(1)$ \\
Hepatitis A & 17 & $1: 20(7)$ & $1: 20(5)$ \\
F. reumatoideo & 12 & $1: 20(5)$ & $1: 40(4)$ \\
Salmonelosis & 14 & $1: 20(4)$ & $1: 40(5)$ \\
Dengue & 15 & $1: 20(1)$ & $1: 40(1)$ \\
Sífilis & 11 & $1: 10(11)$ & $1: 20(6)$ \\
TOTAL & 96 & - & - \\
\hline
\end{tabular}

a valor de referencia para IFI leptospirosis: título mayor o igual a 1:40

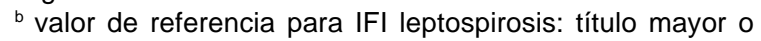
igual a 1:80

La evaluación para obtener los patrones de especificidad frente a otras etiologías producidas por espiroquetas diferentes a Leptospira se hizo con sueros positivos para sífilis; aquí se obtuvieron reacciones cruzadas a una dilución 1:10, pero no a diluciones mayores (cuadro 1).

En forma paralela, al realizar un estudio exploratorio en 27 muestras que habían sido remitidas para diagnóstico de diferentes síndromes febriles, se encontró que el $11 \%$ eran positivas por IFI para anticuerpos contra leptospirosis tipo IgM en títulos hasta de 1:320.

\section{Discusión}

El diagnóstico de la leptospirosis es difícil y son muchas las enfermedades con manifestaciones clínicas similares. El laboratorio es necesario para confirmar el diagnóstico de leptospirosis y para apoyar las acciones de control y de prevención. EI MAT es la prueba de referencia de la OMS y aumenta su confiabilidad cuantos más serovares de referencia y aislamientos locales se utilicen en la reacción $(1,23)$.

En el país, además de la prueba de MAT, algunos laboratorios realizan pruebas serológicas comerciales tipo ELISA u otras. En Colombia es frecuente usar estas pruebas para el diagnóstico serológico de leptospirosis sin un estudio de validación que los respalde. Cuando éste es el 
caso, el clínico debe entender que estas pruebas pueden tener valores de sensibilidad y especificidad que varían de acuerdo a la población y al tipo de antígeno empleado. Cabe anotar que en países donde estas evaluaciones se han llevado a cabo, los rangos de sensibilidad para diferentes fases de la enfermedad van desde el 25 hasta el $92 \%$ para diferentes pruebas comerciales $(10,17)$. En un estudio realizado en el Valle, que comparó las técnicas serológicas PanBio IgM ELISA y MAT en el diagnóstico de leptospirosis, se obtuvo un valor de sensibilidad del 29\% para PanBio (Astudillo H.M, Parra B, Corral R, Barona G, Muñoz E.P, Rengifo G. Comparación de las técnicas serológicas PanBio IgM ELISA y microaglutinacion (MAT) en el diagnóstico de leptospirosis. Biomédica 2003;23 (Suppl.1):86).

Al compararla con el MAT, la prueba de IFI tiene la ventaja de no requerir el mantenimiento de un gran cepario en continuo repique, posibilidad que se restringe a laboratorios de referencia. Además, por utilizar una cepa no patógena de Leptospira, se reduce el riesgo de infección ocupacional entre el personal del laboratorio que la ejecuta. La evaluación de esta prueba de IFI muestra que tiene una sensibilidad del $89,47 \%$ y una especificidad del $100 \%$ para este grupo de estudio específicamente. Las investigaciones de otros grupos de estudio han encontrado que la prueba tiene una sensibilidad de 40,48 y $89,2 \%$ y una especificidad de 85,97 y $100 \%(10,13,16,18)$. Los niveles variables de sensibilidad de la prueba pueden deberse a las diferencias en los antígenos utilizados y a las diferentes fases de la enfermedad en que se encontraban los pacientes cuando les fue tomada la muestra. Los datos de especificidad son comparables con los de los estudios referenciados, coincidiendo en que la IFI es una prueba específica, lo que es ventajoso para el clínico en el momento de definir el diagnóstico de una enfermedad con síntomas tan inespecíficos.

En el caso del grupo de muestras serológicas positivas, dos pacientes no presentaron anticuerpos anti-Leptospira detectados por IFI, lo que puede explicarse porque la muestra fue tomada en la fase convaleciente, cuando los anticuerpos género específicos no estaban circulando sino los serovar específicos, que fueron detectados por MAT y no por IFI (15).

La IFI es una prueba género específica que puede usarse como prueba de tamizaje de la infección antigua por la detección de lgG y como prueba de diagnóstico de la infección reciente monitorizando IgM. Los anticuerpos género específicos aparecen más temprano que los anticuerpos serovar específicos (1), lo que resulta ventajoso, especialmente en pruebas que detectan IgM, y útil para el diagnóstico de la enfermedad, facilitando el inicio oportuno del tratamiento para así evitar complicaciones. Es recomendable entonces que el clínico remita a un diagnóstico serológico por IFI ante la sospecha inicial de un caso de leptospirosis con el fin de aumentar la posibilidad de registrar los anticuerpos género específicos que la prueba detecta y así aumentar su sensibilidad. Sin embargo, se debe disponer de mayor número de pacientes para complementar estos hallazgos y obtener datos que garanticen la validación de la prueba.

Al probar otras enfermedades con agentes etiológicos muy distintos como malaria, hepatitis $B$ y hepatitis $A$, salmonelosis, dengue y pacientes con factor reumatoideo positivo se obtuvieron reacciones no específicas en un número reducido de muestras y en títulos muy bajos (1:20). Sólo se encontró reacción cruzada con sífilis en diluciones por debajo de 1:10 debido a que se comparten antígenos con otras espiroquetas (13).

El estudio exploratorio en el que utilizamos la prueba de IFI como tamiz para determinar la presencia de leptospirosis en muestras remitidas para diagnóstico de síndromes febriles nos indica que ésta es una etiología frecuente que se debe buscar para diferenciar leptospirosis de otras enfermedades febriles agudas. Por ejemplo, se ha documentado ampliamente la asociación de leptospirosis y dengue en diferentes regiones del mundo, algunas veces asociada con brotes después de desastres naturales (25-29). En nuestro medio se ha documentado concomitancia entre dengue y leptospirosis en pacientes con síndrome febril. En Córdoba se registró $4 \%$ de asociación entre dengue y leptospirosis y $2 \%$ entre salmonelosis, dengue y leptospirosis (Miranda JL, 
Conde E, Sánchez I, Martínez P, Ríos R, Gainer $A$, et al. Estudio de un brote de fiebre de origen desconocido en el municipio de Chimá. Biomédica 2003;23 (Suppl. 1):195). En el Valle del Cauca, el $25 \%(106 / 419)$ de los pacientes presentaban infección aguda por virus del dengue y Leptospira. La mayoría de los casos de coinfección correspondió a pacientes con manifestaciones graves de la enfermedad (Parra B, Astudillo M, Corral RH, Muñoz EP, Barona G, Rengifo G. Infecciones mixtas por virus del dengue y Leptospira en pacientes con enfermedad moderada a grave en el Valle del Cauca. Biomédica 2003;23 (Suppl 1):201).

En Colombia es poco lo que se ha informado sobre leptospirosis en humanos, o sobre su comportamiento epidemiológico y serológico (20,30-36). Lo anterior se debe posiblemente a que no existen pruebas serológicas de amplia distribución para confirmar el diagnóstico etiológico. Pocos laboratorios del país tienen los cultivos de cepas patrones de las serovariedades de Leptospira necesarias para el diagnóstico serológico MAT recomendado por la OMS. EI personal clínico debe documentarse sobre cuáles métodos están disponibles en su región.

Este estudio permitió establecer los parámetros de sensibilidad, especificidad y valores predictivos de la prueba de IFI para leptospirosis en grupos de estudio clínico. Así evaluada, está disponible para utilizarla como complemento del diagnóstico de rutina de la enfermedad con la prueba MAT y para estudios seroepidemiológicos.

\section{Conflicto de intereses}

Ninguno.

\section{Financiación}

Esta investigación fue financiada por la Dirección Seccional de Salud de Antioquia y el Instituto Colombiano de Medicina Tropical-CES.

\section{Referencias}

1. World Health Organization. International Leptospirosis Society. Human leptospirosis: guidance for diagnosis, surveillance and control 2003. [Consultado: 05/05/2005]. Disponible en: http:// www.med.monash.edu.au/microbiology/staff/adler/ ilspage.html
2. Bharti AR, Nally JE, Ricaldi JN, Matthias MA, Diaz MM, Lovett MA, et al. Peru-United States Leptospirosis Consortium. Leptospirosis: a zoonotic disease of global importance. Lancet Infect Dis 2003;3:757-71.

3. Meites E, Jay MT, Deresinski S, Shieh WJ, Zaki SR, Tompkins L, et al. Reemerging leptospirosis, California. Emerg Infect Dis 2004;10:406-12.

4. Yasuda PH, Steigerwalt AG, Sulzer KR, Kaufmann AF, Royers F, Brenner DJ. Deoxyribonucleic acid relatedness between serogroups and serovars in the family Leptospiraceae with proposals for seven new Leptospira species. Int J Syst Bacteriol 1987;37:40715.

5. Ramadass P, Jarvis BD, Corner RJ, Penny D, Marshall RB. Genetic characterization of pathogenic Leptospira species by DNA hybridization. Int J Syst Bacteriol 1992;42:215-9.

6. National Center for Biotechnology Information. Taxonomy 2003. [Consultado: 18/05/2005]. Disponible en: http://www.ncbi.nlm.nih.gov/Taxonomy/Browser/ wwwtax.cgi?id=171.

7. Organización Panamericana de la Salud. Definiciones de caso: dengue y leptospirosis. Bol Epidemiol 2000;21. [Consultado: 05/05/2005]. Disponible en: http://www.paho.org/Spanish/SHA/ be_v2n2-casos.htm.

8. Farrar WE. Leptospira species (leptospirosis). En: Mandel GL, Bennett JE, Dolin R, editors. Principles and practice of infectious diseases. New York: Churchill Livingstone;1995. p.2137-41.

9. de Silva MV, Nakamura PM, Camargo ED, Batista L, Vaz AJ, Romero EC, et al. Immunodiagnosis of human leptospirosis by Dot-ELISA for the detection of IgM, IgG and IgA antibodies. Am J Trop Med Hyg 1997;56:650-5.

10. Effler PV, Bogard AK, Domen HY, Katz AR, Higa HY, Sasaki DM. Evaluation of eight rapid screening tests for acute leptospirosis in Hawaii. J Clin Microbiol 2002;40:1464-9.

11. Levett PN, Branch SL. Evaluation of two enzymelinked immunosorbent assay methods for detection of immunoglobulin $\mathrm{M}$ antibodies in acute leptospirosis. Am J Trop Med Hyg 2002;66:745-8.

12. Levett PN. Usefulness of serologic analysis as a predictor of the infecting serovar in patients with severe leptospirosis. Clin Infect Dis 2003,36:447-52.

13. Appassakij H, Silpapojakul K, Wansit R, Woodtayakorn J. Evaluation of the immunofluorescent antibody test for the diagnosis of human leptospirosis. Am J Trop Med Hyg 1995;52:340-3.

14. Sehgal SC, Vijayachari P, Sharma S, Sugunan AP. LEPTO Dipstick: a rapid and simple method for serodiagnosis of acute leptospirosis. Trans R Soc Trop Med Hyg 1999;93:161-4. 
15. Cumberland P, Everard CO, Levett PN. Assessment of the efficacy of an IgM-Elisa and microscopic agglutination test (MAT) in the diagnosis of acute leptospirosis. Am J Trop Med Hyg 1999;61:731-4.

16. Joshi S, Bal A, Bharadwaj R, Kumbhar R, Kagal A, Arjunwadkar V. Role of $\operatorname{lgM}$ specific indirect immunofluorescence assay in diagnosing an outbreak of leptospirosis. Indian J Pathol Microbiol 2002;45:757.

17. Bajani MD, Ashford DA, Bragg SL, Woods CW, Aye T, Spiegel RA, et al. Evaluation of four commercially available rapid serologic tests for diagnosis of leptospirosis. J Clin Microbiol 2003;41:803-9.

18. Pradutkanchana S, Pradutkanchana J, Khuntikij P. Detection of $\operatorname{lgM}$ specific antibody using indirect immunofluorescent assay for diagnosis of acute leptospirosis. J Med Assoc Thai 2003;86:641-6.

19. Russell KL, Montiel Gonzalez MA, Watts DM, Lagos-Figueroa RC, Chauca G, Ore M, et al. An outbreak of leptospirosis among Peruvian military recruits. Am J Trop Med Hyg 2003;69:53-7.

20. Sebek Z, Sixl W, Valova M, Marth E, Dock M, Reinthaler FF. Serological investigations for leptospirosis in humans in Columbia. Geogr Med Suppl 1989;3:51-60.

21. Céspedes M. Manual de procedimientos bacteriológico y serológico para el diagnóstico de la leptospirosis. Serie de Normas Técnicas No. 34. Lima: Ministerio de Salud del Perú. Instituto Nacional de Salud; 2002. p.53.

22. Katz AR, Effler PV. "Probable" versus "confirmed" leptospirosis: an epidemiologic and clinical comparison utilizing a surveillance case classification. Ann Epidemio 2003;13:196-203.

23. Faine S. Guide pour la lutte contre la leptospirose. Publicación Offset No. 67. Geneve: Organización Mundial de la Salud; 1987. p. 50.

24. Riegelman RK, Hirsch RP. Cómo estudiar un estudio y probar una prueba: lectura crítica de la literatura médica. $2^{\mathrm{a}}$ ed. Publicación científica 531. Washington D.C.: OPS; 1992
25. Levett PN, Branch SL, Edwards CN. Detection of dengue infection in patients investigated for leptospirosis in Barbados. Am J Trop Med Hyg 2000;62:112-4.

26. Sanders EJ, Rigau-Perez JG, Smits HL, Deseda CC, Vorndam VA, Aye T, et al. Increase of leptospirosis in dengue-negative patients after a hurricane in Puerto Rico in 1996 [correction of 1966]. Am J Trop Med Hyg 1999;61:399-404.

27. Flannery B, Pereira MM, Velloso L de F, Carvalho C de C, de Codes LG, Orrico G de S, et al. Referral pattern of leptospirosis cases during a large urban epidemic of dengue. Am J Trop Med Hyg 2001;65:65763.

28. Hossain MA, Khatun M, Arjumand F, Nisaluk A, Breiman RF. Serologic evidence of dengue infection before onset of epidemic, Bangladesh. Emerg Infect Dis 2003;9:1411-4.

29. LaRocque RC, Breiman RF, Ari MD, Morey RE, Janan FA, Hayes JM, et al. Leptospirosis during dengue outbreak, Bangladesh. Emerg Infect Dis 2005;11:766-9.

30. Bravo C, Restrepo M. Leptospirosis en Antioquia. II. Leptospirosis en perros y cerdos. Antioquia Médica 1970;20:19-23.

31. Acosta $\mathbf{H}$, Moreno $\mathbf{C H}$, Viáfara D. Leptospirosis. Colombia Médica 1994;25:36-42.

32. Epsein PR, Calix Pena O, Blanco Racedo J. Climate and disease in Colombia. Lancet 1995;346:1243-4.

33. Perez-Garcia J. Hallazgos histopatológicos en necropsias de leptospirosis. Colombia Médica 1997;28:4-9.

34. Hoyos JA, Arango JH, De Lima E. Leptospirosis icterohemorrágica. Colombia Médica 1998;29:43-6.

35. Ochoa JE, Sanchez A, Ruiz I. Epidemiología de la leptospirosis en una zona andina de producción pecuaria. Rev Panam Salud Publica 2000;7:325-31.

36. Najera S, Alvis N, Babilonia D, Alvarez L, Mattar S. Occupational leptospirosis in a Colombian Caribbean area. Salud Publica Mex 2005;47:240-4. 